\title{
CONVECCIÓN DE CALOR EN EL FLUJO DE FLUIDOS A TRAVÉS DE UN LECHO DE EMPAQUE DE BAUXITA ACTIVADA
}

\author{
Luis Patiño $^{1}$ Rosmely Figueroa ${ }^{1}$ \\ Recibido el 9 de marzo de 2004, aceptado el 12 de Noviembre de 2004
}

\begin{abstract}
RESUMEN
Se presenta una metodología teórico-experimental para determinar los coeficientes intersticiales de transferencia de calor promedio en el flujo de agua a través de un lecho de empaque de bauxita activada donde el fluido no está en equilibrio térmico con la fase sólida. Los coeficientes de transferencia de calor se obtienen a través del "Single Blow Transient Method" combinando los resultados experimentales obtenidos en un banco de ensayo con las soluciones numéricas del modelo matemático El sistema de ecuaciones diferenciales parciales generado en el modelo matemático es resuelto a través de un procedimiento numérico basada en el método de volúmenes finitos. Las pruebas experimentales y las soluciones numéricas se realizaron para diferentes valores de velocidad superficial del fluido a la entrada del lecho y para diferentes valores de porosidad del medio, obteniéndose que los números de Nusselt dependen en gran escala de los números de Reynolds y la porosidad del medio.
\end{abstract}

Palabras claves: Convección de calor; medios porosos, bauxita activada.

\begin{abstract}
A theoretic-experimental methodology is presented to determine the interstitial heat transfer coefficients in the water flow through packed bed of activated Bauxite, where the fluid is not in heat balance with the solid phase. The heat transfer coefficients are obtained through "Single Blow Transient Method" combining the experimental results obtained in a test set-up with the numerical solutions of the mathematical model. The system of partial differential equations generated in the mathematical model is solved through a numerical procedure based on the finite volumes method. Experimental test results and numerical solutions were obtained for different values of superficial speed of the fluid from the entrance of the bed and for different porosity values, obtaining themselves that the Nusselt numbers depend in great scale on the Reynolds numbers and the porosity of media.
\end{abstract}

Keywords: Heat convection, porous media, activated bauxite.

\section{INTRODUCCIÓN}

Durante muchos años se han venido desarrollando investigaciones en el área de la transferencia de calor a través de medios porosos, en vista de las innumerables aplicaciones industriales que tiene. Entre éstas se pueden citar la ingeniería de yacimientos de agua (acuíferos), de petróleo y de gas, diseño de reactores químicos, sistemas geofísicos y geotérmicos, diseño de intercambiadores de calor tipo regeneradores, diseño de procesos de separación (como por ejemplo la adsorción), entre otros. La Adsorción es un proceso mediante el cual se hace pasar un fluido a través de un recipiente que contiene un lecho fijo (medio poroso) de naturaleza adsorbente. En este fenómeno se presenta transferencia de masa en régimen transitorio desde una fase fluida hasta otra sólida, y presenta transferencia de calor entre las fases si la temperatura del fluido es diferente a la temperatura de la matriz porosa (adsorción no isotérmica). Este tipo de proceso se aplica en sistemas donde se quiera obtener separación de productos, purificación de diluentes, recuperación de solutos y decoloración de diluentes. Entre algunos de los materiales que se utilizan como adsorbentes se mencionan la alúmina, tierra infusoria, arcilla, carbón activado, óxido de silicio, bauxita activada, etc. La bauxita activada es un mineral que es muy utilizado en procesos de adsorción no isotérmica y consiste principalmente de Alúmina $\left(\mathrm{Al}_{2} \mathrm{O}_{3}\right)$, y en menor proporción de bióxido de silicio $\left(\mathrm{SiO}_{2}\right)$, bióxido de titanio $\left(\mathrm{TiO}_{2}\right)$ y óxido de hierro $\left(\mathrm{Fe}_{2} \mathrm{O}_{3}\right)$.

\footnotetext{
${ }^{1}$ Universidad de Oriente, Departamento de Mecánica, Postal 4653, Puerto La Cruz, Venezuela, lpatino@udo.edu.ve
} 


\section{EQUIPO Y PROCEDIMIENTO EXPERIMENTAL}

\section{Equipo experimental}

La Fig. 1 muestra una representación esquemática del equipo utilizado para realizar las pruebas experimentales. El mismo consta fundamentalmente de cuatro partes principales que se describen a continuación:

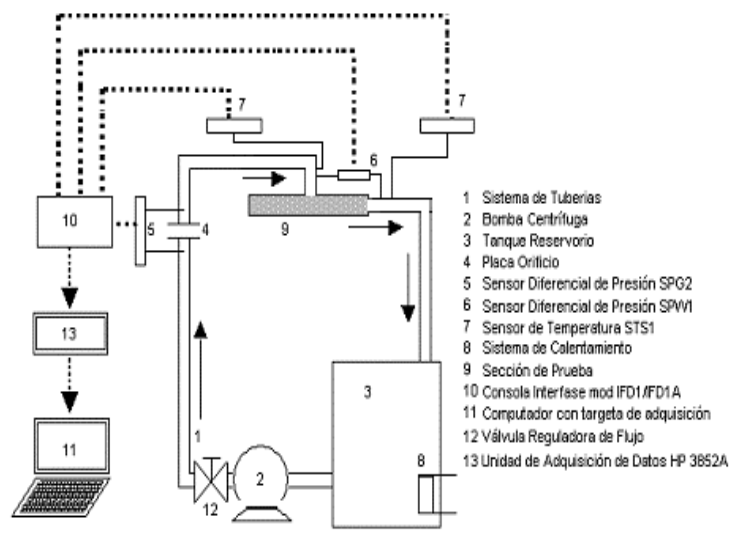

Fig. 1 Esquema del banco experimental.

Sistema de bombeo y transporte de líquido: Está constituido por una bomba centrífuga de $1 / 2$ HP que transporta el agua por medio de un sistema de tuberías de acero de 3/4" de diámetro desde un tanque de almacenamiento hasta otro receptor, pasando a través de la sección de pruebas. El fluido en el tanque de almacenamiento es calentada hasta una temperatura deseada por medio de un par de resistencias eléctricas de 1200 vatios.

Sección de pruebas: Está constituida por un conducto de acero de sección transversal constante y circular de 2" de diámetro y una longitud de $22,5 \mathrm{~cm}$, conectada al sistema de tuberías por intermedio de bridas. Esta sección se encuentra aislada térmicamente. Es importante destacar que en el interior de esta sección se encuentra colocado el medio poroso.

Medio poroso: Para modelar el medio poroso se utilizaron esferas de bauxita activada, cuya composición es $62,3 \%$ en peso de $\mathrm{Al}_{2} \mathrm{O}_{3}, 9,46 \%$ en peso de $\mathrm{SiO}_{2}$, $2,89 \%$ en peso de $\mathrm{TiO}_{2}, 5,9 \%$ en peso de $\mathrm{Fe}_{2} \mathrm{O}_{3}$ y 14,2 $\%$ en peso de volátiles. La bauxita activada presenta valores de densidad de $3200 \mathrm{Kg} / \mathrm{m}^{3}$, conductividad térmica $31 \mathrm{~W} / \mathrm{mK}$ y calor específicico $795 \mathrm{~J} / \mathrm{KgK}$. La porosidad del medio fue evaluada utilizando la expresión $\phi=\left(\mathrm{v}_{\mathrm{t}}-\mathrm{v}_{\mathrm{s}}\right) / \mathrm{v}_{\mathrm{t}}$. En esta expresión $\mathrm{v}_{\mathrm{t}}$ es el volumen total de la sección de pruebas y $\mathrm{v}_{\mathrm{s}}$ el volumen ocupado por el sólido. La estrategia planteada para generar medios porosos con diferentes porosidades fue utilizar distintos rangos de diámetros de partículas esféricas en cada una de las pruebas. Los detalles de la granulometría y porosidades utilizadas se presentan en la Tabla 1.

Sistema de adquisición de datos: Está constituido por un sensor diferencial de presión ubicado en la placa orificio (cuya función es determinar el flujo volumétrico de agua), y dos sensores de temperatura ubicados justamente en la entrada y la salida de la sección de pruebas. Todos estos medidores están interconectados a una unidad de adquisición de datos (HP3852A). Los datos adquiridos son configurados y leídos por un computador PC gracias a un programa computacional de adquisición elaborado en el lenguaje de programación HP-Basic.

\section{Procedimiento Experimental}

Los experimentos se realizaron variando la velocidad del fluido a la entrada de la sección de pruebas y variando también la porosidad del medio. Se realizaron cincuenta corridas experimentales; diez corridas, con diferentes flujos volumétricos (números de Reynolds) para cada porosidad. Para mayores detalles el lector puede consultar la referencia [12].

\section{MODELO MATEMÁTICO DEL PROCESO}

El problema en estudio consiste en la determinación de los coeficientes fílmicos de transferencia de calor promedio en el flujo de fluidos (agua) a través de un lecho de empaque de bauxita activada, donde el fluido no está en equilibrio térmico con la fase sólida. En esta sección se indica la formulación matemática que permite modelar la convección de calor transitoria en el flujo de fluidos a través de medios porosos, y la conducción de calor en el sólido. Este modelo cuenta con ecuaciones que se derivan realizando los balances de conservación de masa, momento y energía en fase fluida, y un balance de energía en la fase sólida. Todos los detalles referentes a la deducción de la presente formulación matemática se encuentran en la referencia [12].

\section{Ecuaciones Gobernantes}

Tomando como fluido en estudio, agua en estado líquido transportándose a través de la sección de pruebas (lecho de empaque), suponiendo que el líquido es incompresible y newtoniano donde las propiedades tales como densidad $(\rho)$, viscosidad absoluta $(\mu)$, 
conductividad térmica (k) y calor específico (Cp) permanecen constantes. Considerando que la lecho de empaque está constituida por esferas de bauxita activada, y que es isotrópica, homogénea e indeformable cuyas propiedades tales como porosidad $(\varepsilon)$, permeabilidad $(\mathrm{K})$, densidad $\left(\rho_{\mathrm{s}}\right)$, conductividad térmica $\left(\mathrm{k}_{\mathrm{s}}\right)$ y calor específico $\left(\mathrm{C}_{\mathrm{s}}\right)$ son constantes, las ecuaciones de variación en coordenadas cilíndricas y régimen transitorio, pueden expresarse como:

Ecuación de Continuidad:

$$
\begin{aligned}
& \frac{\partial}{\partial t}(\rho v)+\frac{\rho}{\varepsilon} v \frac{\partial v}{\partial r}+\frac{\rho}{\varepsilon} u \frac{\partial v}{\partial z}=-\varepsilon \frac{\partial p}{\partial r}+ \\
& \mu\left[\frac{\partial}{\partial r}\left(\frac{1}{r} \frac{\partial}{\partial r}(r v)\right)+\frac{\partial^{2} v}{\partial z^{2}}\right]-\frac{\mu}{K} \varepsilon v-\frac{\rho F \varepsilon}{\sqrt{K}} \cdot|\underline{V}| \cdot v
\end{aligned}
$$

Donde u es la velocidad del fluido en la dirección axial $(z), \mathrm{v}$ es la velocidad del fluido en la dirección radial $(r)$ y $t$ es el tiempo

Ecuación de Cantidad de Movimiento en la Dirección Axial:

$$
\begin{aligned}
& \rho\left(\frac{\partial u}{\partial t}+(1 / \varepsilon) v \frac{\partial u}{\partial r}+(1 / \varepsilon) u \frac{\partial u}{\partial z}\right)=-\varepsilon \frac{\partial p}{\partial z}+ \\
& \mu\left[\frac{1}{r} \frac{\partial}{\partial r}\left(r \frac{\partial u}{\partial r}\right)+\frac{\partial^{2} u}{\partial r^{2}}\right]-\frac{\mu \varepsilon}{K} u-\frac{\rho F \varepsilon}{\sqrt{K}}|V| \cdot u
\end{aligned}
$$

Donde $p$ es la presión del fluido; $\underline{V}$ es el vector velocidad del fluido; $F$ es el coeficiente inercial y también se le denomina el término de Forchheimer [13]; y $K$ es la permeabilidad del medio.

Ecuación de Cantidad de Movimiento en la Dirección Radial:

Ecuación de la Energía de la Fase Líquida:

$$
\begin{gathered}
\rho\left(\frac{\partial u}{\partial t}+(1 / \varepsilon) v \frac{\partial u}{\partial r}+(1 / \varepsilon) u \frac{\partial u}{\partial z}\right)=-\varepsilon \frac{\partial p}{\partial z}+ \\
\mu\left[\frac{1}{r} \frac{\partial}{\partial r}\left(r \frac{\partial u}{\partial r}\right)+\frac{\partial^{2} u}{\partial r^{2}}\right]-\frac{\mu \varepsilon}{K} u-\frac{\rho F \varepsilon}{\sqrt{K}}|V| \cdot u \\
\varepsilon \rho C p\left(\frac{\partial T}{\partial t}+(1 / \varepsilon) v \frac{\partial T}{\partial r}+(1 / \varepsilon) u \frac{\partial T}{\partial z}\right)+ \\
h A\left(T-T_{S}\right)=\frac{1}{r} \frac{\partial}{\partial r}\left(k \varepsilon r \frac{\partial T}{\partial r}\right)+\frac{\partial}{\partial z}\left(k \varepsilon \frac{\partial T}{\partial z}\right)
\end{gathered}
$$

Donde $T$ es la temperatura en la fase líquida; $T s$ es la temperatura en la fase sólida; $h$ es el coeficiente intersticial de convección de calor; y $A$ es el área superficial de transferencia de calor.

Ecuación de la Energía de la Fase Sólida:

$$
\begin{aligned}
& \rho_{s} C p_{s}(1-\varepsilon) \frac{\partial T_{s}}{\partial t}+h A\left(T_{s}-T\right)= \\
& \frac{1}{r} \frac{\partial}{\partial r}\left(k_{s}(1-\varepsilon) r \frac{\partial T}{\partial r}\right)+\frac{\partial}{\partial z}\left(k_{s}(1-\varepsilon) \frac{\partial T}{\partial z}\right)
\end{aligned}
$$

Es importante indicar que la permeabilidad del medio y el coeficiente inercial son calculados a través de las ecuaciones (6) [14]. En las ecuaciones (6) $d_{p}$ representa el diámetro promedio de la partícula que conforma el medio poroso.

$$
K=\frac{\varepsilon^{3} d p^{2}}{150(1-\varepsilon)^{2}}, \quad F=\frac{1.75}{\sqrt{150} \varepsilon^{\frac{3}{2}}}
$$

\section{Condiciones del Problema}

Las condiciones iniciales son velocidades radiales y axiales nulas, y temperaturas homogéneas para las fases líquidas y sólidas (Ti). Las condiciones de borde asociadas al problema son: Las velocidades u y v en las paredes son nulas. La velocidad u es máxima y la velocidad v es nula en el eje de simetría. En la entrada la velocidad axial $\left(\mathrm{u}_{0}\right)$ es uniforme, la velocidad $\mathrm{v}$ es nula $\mathrm{y}$ la temperatura $\left(\mathrm{T}_{0}\right)$ en el líquido es prescrita, uniforme y variable en el tiempo. En el eje de simetría el flujo de calor por conducción es nulo. En la salida se tienen condiciones localmente parabólicas. Las condiciones de borde térmicas para la fase sólida son iguales a la de la fase fluida.

\section{SOLUCIÓN DEL MODELO MATEMÁTICO}

Para la resolución de este modelo se empleó una metodología numérica. Las ecuaciones diferenciales con sus respectivas condiciones de borde e iniciales descritas en el modelo matemático fueron resueltas a través del código comercial CFX versión 4.3, el cual basa la solución en el método de los Volúmenes Finitos [15]. Para todas las simulaciones los flujos convectivos y difusivos en las interfaces de los volúmenes se calculan a través del esquema de interpolación híbrido y el procedimiento de acoplamiento Presión - Velocidad, en las ecuaciones de movimiento, utilizado fue el SIMPLE Revista Facultad de Ingeniería, Chile, Vol.12 No2, 2004 
[16]. El régimen transitorio fue implementado a través de un esquema totalmente implícito y el criterio de convergencia fue establecido tomando el máximo residuo en la ecuación de continuidad. La temperatura inicial en cada simulación dependió de los resultados experimentales obtenidos, y están en un rango entre 25 y $35^{\circ} \mathrm{C}$. Para discretizar el dominio (sección de pruebas) y para definir el tamaño del incremento de tiempo, se realizaron dos análisis de sensibilidad, los cuales arrojaron un tamaño de malla de 80 volúmenes en la dirección radial y 24 en la dirección axial, lo cual generó una discretización de 1920 volúmenes finitos, y un incremento de tiempo de 0,1 segundos.

\section{COEFICIENTES INTERSTICIALES DE TRANSFERENCIA DE CALOR}

Para determinar los coeficientes intersticiales de convección de calor "h" para cada una de las corridas experimentales se utilizó el "single blow transient method" [11]. Este método necesita únicamente los perfiles transitorios promedio de temperatura del fluido a la entrada y a la salida de la sección de pruebas, y consiste en suponer un valor de prueba para h, calculando seguidamente, a través del código CFX, el perfil transitorio de temperatura y verificando que la temperatura a la salida calculada coincida, con un error menor a $10^{-3}$ con la temperatura a la salida experimental. Este proceso se repite hasta obtener seis casos en que se cumpla el error. El coeficiente h, será el promedio aritmético de los coeficientes h para los seis casos. La Fig. 2 muestra un flujograma descriptivo del Single Blow Transient Method como una técnica para determinar los coeficientes de transferencia de calor en problemas en estado transitorio.

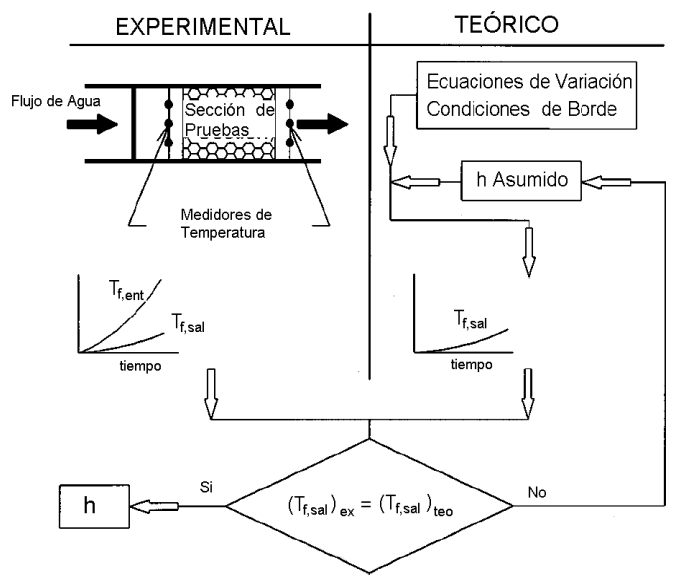

Fig 2 Diagrama de flujo representativo del Single Blow Transient Method.

\section{ANÁLISIS DE INCERTIDUMBRE}

Los errores en el cálculo de los coeficientes de transferencia de calor son producidos fundamentalmente por las desviaciones en las mediciones de los flujos volumétricos, las mediciones de las temperaturas y la raíz cuadrada de las diferencias medias de temperatura entre los valores experimentales y los calculados numéricamente. En esta investigación se utilizó el método de incertidumbre desarrollado por Kline $\mathrm{y}$ McClintock [17] y se obtuvo un valor de incertidumbre para el flujo másico de $0.5 \%$, para las mediciones de temperatura de $3.2 \%$, y para la raíz cuadrada de las diferencias medias de temperatura de $5.1 \%$. Como resultado de esto se obtuvo una incertidumbre global del coeficiente de convección de calor de $5.1 \%$.

\section{ANÁLISIS DE RESULTADOS}

Se realizaron cincuenta simulaciones numéricas que modelan el intercambio de energía térmica entre el fluido y el medio poroso descrito en esta investigación. Por razones de limitación de espacio solo se presentan las curvas de respuesta de temperatura para algunas de las simulaciones realizadas.

En la Fig. 3 se muestran los perfiles de temperaturas del fluido a la entrada y a la salida de la sección de pruebas obtenidos experimentalmente, y se comparan con el perfil transitorio del fluido a la salida de la sección determinado teóricamente a través del modelo numérico y del Single Blow Transient Method. Adicionalmente en ellas se presenta el valor del coeficiente de convección de calor determinado en dichas pruebas. En forma general se aprecia que existe una muy buena aproximación entre los resultados experimentales y los resultados teóricos con un error máximo de aproximadamente $0.1 \%$. 

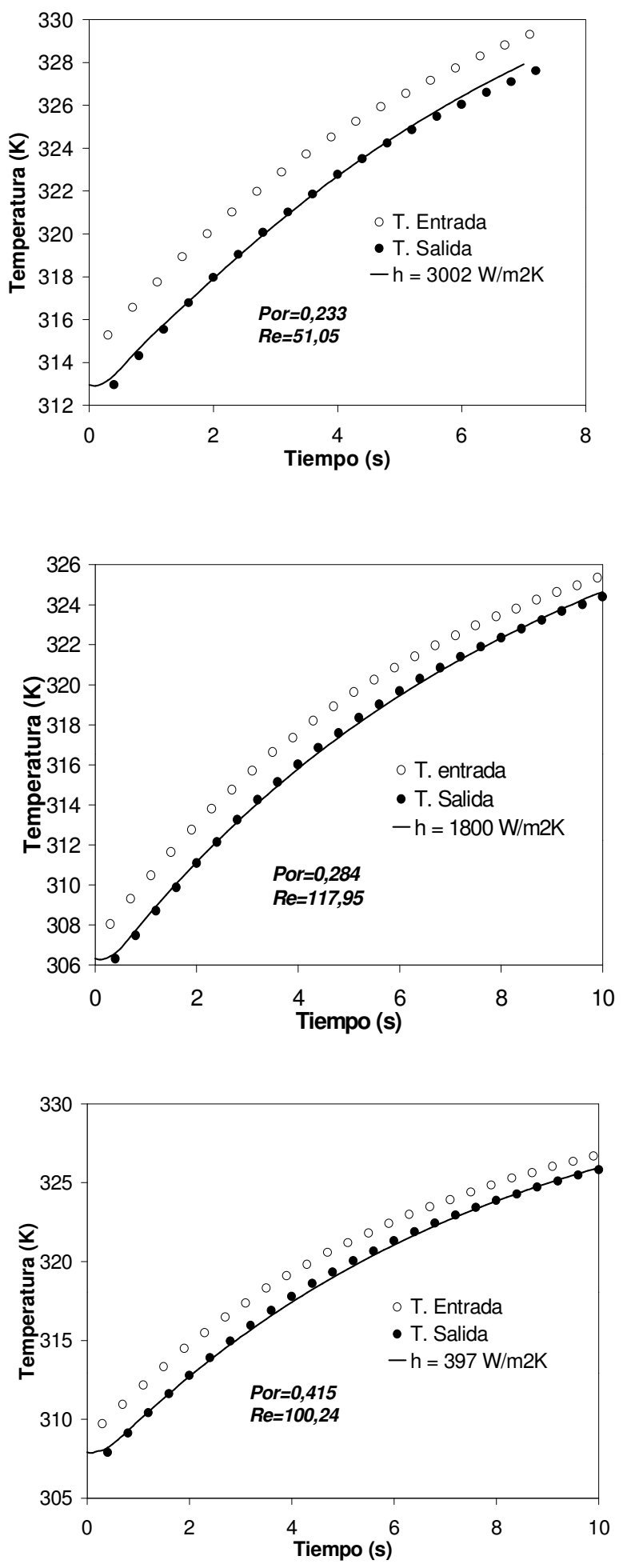

Fig. 3 Perfiles transitorios de temperatura del fluido.
En la Fig. 4 se presentan los perfiles transitorios de temperaturas del fluido y de la matriz sólida para tres posiciones axiales del dominio estudiado y para tres valores de porosidades $0.284,0.34$ y 0.415 respectivamente. Se observa que a medida que transcurre el tiempo la temperatura de cualquier punto en la dirección del flujo va en ascenso. La presencia del medio poroso en el camino del fluido va a generar una disminución en la temperatura de éste. Esta disminución de la energía térmica en el fluido es absorbida por la matriz porosa. A pesar que el sólido está inicialmente a una temperatura uniforme, éste aumenta su temperatura debido al intercambio térmico con la fase fluida. A medida que transcurre el tiempo la temperatura del sólido se incrementa para cualquier posición, sin embargo esta temperatura va en descenso cuando se incrementa la coordenada axial. Esto se debe fundamentalmente al hecho que el sólido se calienta debido al contacto con el fluido. Si el fluido se enfría cuando se desplaza en la dirección del movimiento, entonces, el sólido que está en contacto con él también presentará disminución en su temperatura. Adicionalmente estas figuras evidencian la ausencia del equilibrio térmico entre las dos fases y muestran que a medida que aumenta la porosidad del medio esta diferencia de temperaturas entre fluido y sólido se incrementa, disminuyendo la transferencia de calor entre estas dos fases.

En la Fig. 5 se muestran los perfiles de temperaturas del fluido y del sólido en función de la posición y para dos instantes de tiempo ( 2 y 20 segundos). La parte superior de la figura muestra la distribución de temperaturas del fluido y su respectiva leyenda es la que está ubicada en el lado derecho de la figura. En la parte inferior de la figura se muestra la distribución de temperaturas del sólido y su respectiva leyenda es la que está ubicada en el lado izquierdo de la figura. Para todos los casos se observa que la temperatura de la fase fluida es de magnitud mayor que la de la fase sólida, demostrando la ausencia de equilibrio térmico entre las fases. 

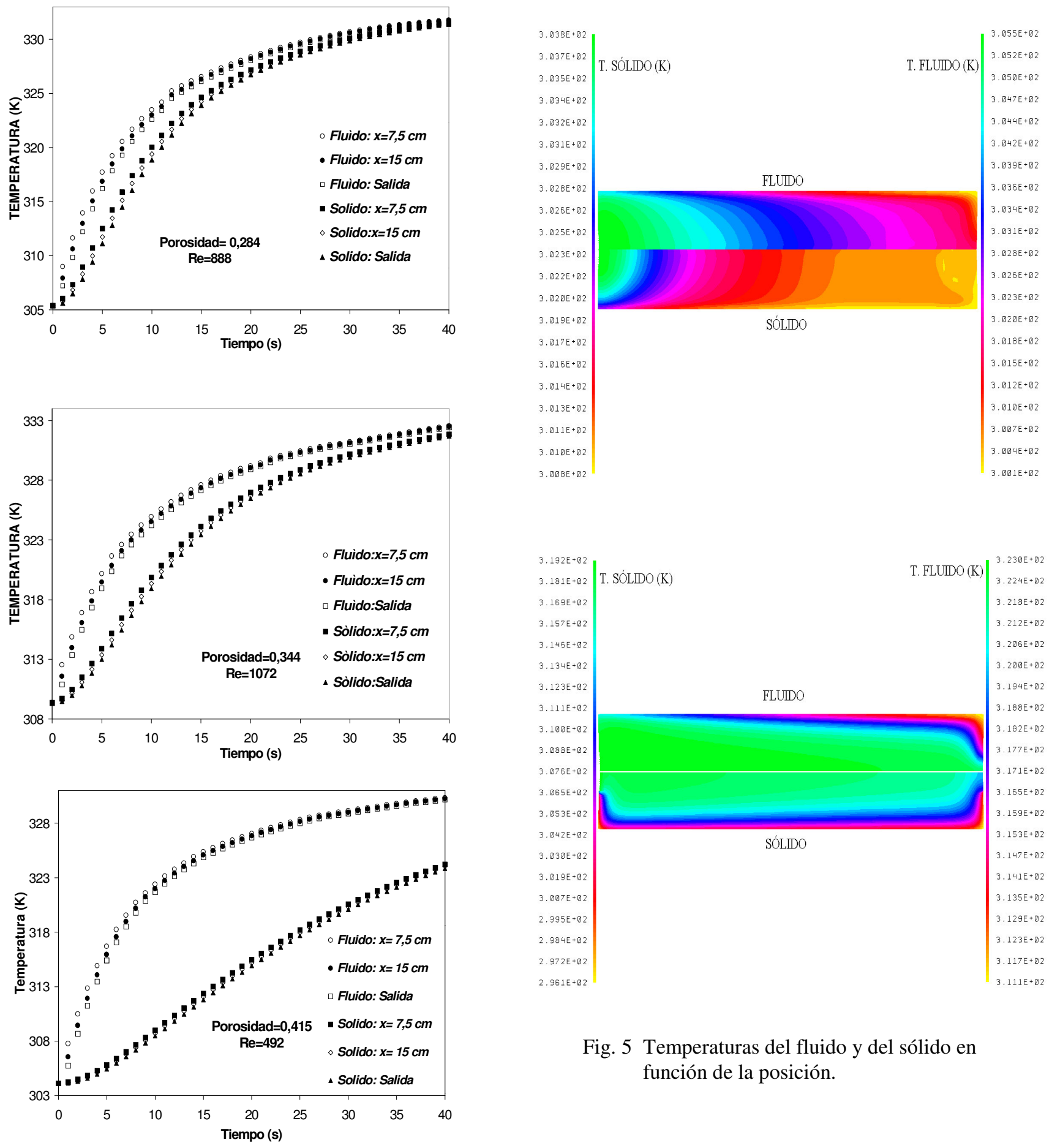

Fig. 5 Temperaturas del fluido y del sólido en función de la posición.

Fig. 4 Perfiles transitorios de temperatura del fluido y de la matriz sólida. 
En la presente investigación las características térmicas del sistema constituido por agua como fluido y esferas de bauxita activada como medio poroso son determinadas a través de la obtención de los números de Nusselt en función de los números de Reynolds y de la porosidad del medio. En la Fig. 6 se presentan los valores de los números de Nusselt vs. el número de Reynolds para los cinco valores de porosidad estudiadas. El número de Nusselt utilizado está basado en una longitud característica igual al diámetro promedio de la partícula que constituye el medio poroso, $\mathrm{Nu}=\mathrm{hd}_{\mathrm{p}} / \mathrm{k}_{\mathrm{f}}$, donde $\mathrm{h}$ es el coeficiente fílmico o intersticial de transferencia de calor. En esta figura se aprecia claramente que el coeficiente de transferencia de calor $\mathrm{h}$ es una variable dependiente tanto de la porosidad del medio, como del número de Reynolds. A medida que aumenta la porosidad del medio, el número de Nusselt disminuye, y a medida que aumenta el número de Reynolds, entonces, el número de Nusselt también lo hace. En base a los resultados obtenidos en la presente investigación, y específicamente a los mostrados en esta última figura, se proponen las siguientes correlaciones empíricas para relacionar los números de Nusselt $\left(\mathrm{Nu}_{\mathrm{d}}\right)$ con los números de Reynolds $\left(\mathrm{Re}_{\mathrm{d}}\right)$ para diferentes porosidades.

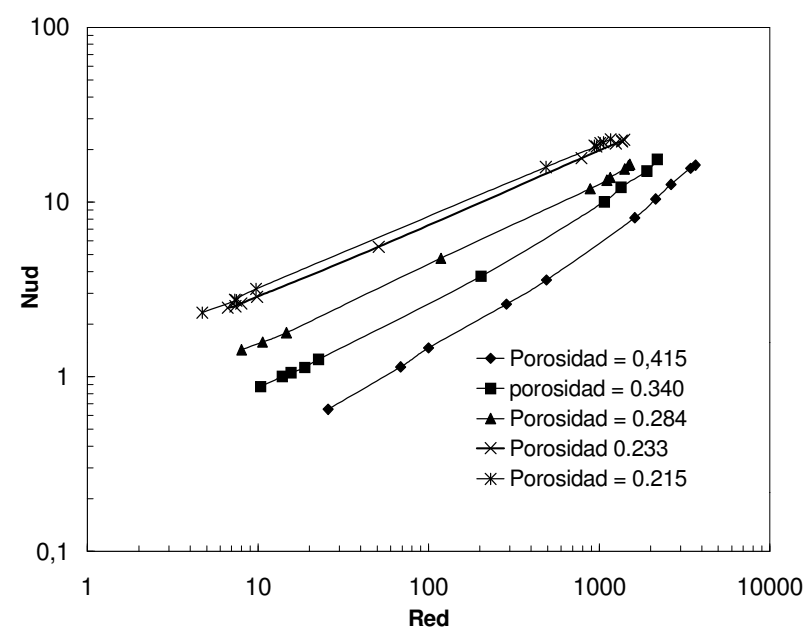

Fig. 6 Número de Nusselt vs. número de Reynolds.

$$
\begin{aligned}
& \mathrm{Nu}_{\mathrm{d}}=1.2119 \mathrm{Re}_{\mathrm{d}}{ }^{0.4151} \text { para porosidad igual a } 0.215 \mathrm{y} \\
& 5<\operatorname{Re}_{\mathrm{d}}<1100 \\
& \mathrm{Nu}_{\mathrm{d}}=1.1076 \mathrm{Re}_{\mathrm{d}}{ }^{0.4163} \text { para porosidad igual a } 0.233 \mathrm{y} \\
& 7<\operatorname{Re}_{\mathrm{d}}<1400
\end{aligned}
$$

$\mathrm{Nu}_{\mathrm{d}}=0.5246 \mathrm{Re}_{\mathrm{d}}{ }^{0.4654}$ para porosidad igual a $0.284 \mathrm{y}$

$$
\begin{aligned}
& 8<\operatorname{Re}_{\mathrm{d}}<1500 \\
& \begin{array}{l}
\mathrm{Nu}_{\mathrm{d}}=0.2288 \mathrm{Re}_{\mathrm{d}}{ }^{0.5511} \\
11<\mathrm{Re}_{\mathrm{d}}<2100
\end{array} \\
& \begin{array}{l}
\mathrm{Nu}_{\mathrm{d}}=0.0718 \mathrm{Re}_{\mathrm{d}}{ }^{0.651} \\
26<\mathrm{Re}_{\mathrm{d}}<3600
\end{array} \quad \text { para porosidad igual a } 0.340 \mathrm{y} \\
&
\end{aligned}
$$

\section{CONCLUSIONES}

Se ha presentado el desarrollo de un modelo matemático y la solución de un problema de convección de calor transitoria en un medio poroso tomando en consideración un dominio axisimétrico y el uso de las velocidades axiales y radiales del fluido para el cálculo del transporte de energía desde el fluido hasta el sólido.

Se evidenció la no existencia del equilibrio térmico entre las fases fluida y sólida, y los perfiles de temperaturas de las fases fluidas y sólida (bauxita activada) dependen de la posición radial y axial, y adicionalmente también dependen del tiempo.

El método Single Blow Transient, conjuntamente con el modelo teórico propuesto, representan una técnica efectiva para la determinación de los coeficientes de transferencia de calor en el flujo de fluidos a través de medios porosos.

Se ha demostrado que el número de Nusselt, y por ende, el coeficiente intersticial de transferencia de calor, en el flujo de fluidos a través de medios porosos dependen tanto del número de Reynolds como de la porosidad del medio. A medida que aumenta el número de Reynolds, el número de Nusselt aumenta, y al aumentar la porosidad del medio, el número de Nusselt disminuye. El lecho de empaque de porosidad 0,215 generaron los mayores números de Nusselt, y el de porosidad 0,415 los menores.

Se han propuesto cinco correlaciones para determinar el número de Nusselt entre agua y un medio poroso de bauxita activada, una para cada valor de porosidad estudiada. Cada una de éstas dependen del número de Reynolds obedeciendo a una ley de la potencia.

\section{AGRADECIMIENTOS}

Los autores desean agradecer a Fundacite-Anzoátegui por el financiamiento brindado para la construcción del equipo experimental mencionado en este artículo. 


\section{REFERENCIAS}

[1] K. Vafai y C. Tien, 'Boundary and Inertia Effects on Flow and Heat Transferin Porous Media". Int. J. Heat Mass Transfer. 24, 6, pp. 195-203, 1981.

[2] K. Vafai, R. Alkire y C. Tien, "An Experime ntal Investigations of Heat Transfer in Variable Porosity Media". Asme Journal Of Heat Transfer. 107, 4, pp. 642-647, 1985.

[3] C. Beckerman and R. Viskanta, 'Forced Convection Boundary Layer Flow and Heat Transfer Along a Flate Plate Embedded in a Porous Media", Int. J. Heat Mass Transfer. 30, 7, pp. 1547-1551, 1987.

[4] P. Cheng y H. Zhu, 'Effects of Radial Thermal Dispersion on Fully Developed Forced Convection in Cylindrical Packed Bed", Int. J. Heat Mass Transfer. 30, 4, pp. 2373-2383, 1987.

[5] A. Shenoy, 'Natural Forced and Mixed Convection Heat Transfer in Non_Newtonian Power- Law Fluid Saturated Porous Media", Transport Of Porous Media. 4, 2, pp. 219-241, 1993.

[6] G. Hwang and C. Chao, 'Heat Transfer measurement and Analytical for sintered Porous Channells", Asme Journal Of Heat Transfer. 116, 3, pp. 456-464, 1994.

[7] G. Hwang, C. Wu and C. Chao, 'Investigation of Non-Darcian Forced Convection in an asymmetrically Heated Sintered Porous Channel", Asme Journal Of Heat Transfer. 117, 4, pp. 725-732 1995.

[8] C. Wu and G. Hwang, "Flow and Heat Transfer Characteristics Inside Packed and Fluidized Beds", Asme Journal Of Heat Transfer. 120, 2, pp. 667-673 1998.

[9] J. Hwang, G. Hwang, R. Yeh and C. Chao, 'Measurement of Interstitial Convective Heat Transfer and Frictional Drag for Flow Across Metal Foams", Asme Journal Of Heat Transfer. 124, 2, pp. 1-10 2002.
[10] C. Howard, 'The Single Blow Problem Including The Effect Of Longitudinal Conduction", presented at The Gas Turbine Conference And Product Show, Asme Paper No. 64-Gt2-11, Houston, Tx, 1964.

[11] C. Liang and W. Yang, 'Modified Single-Blow Technique for Performance Evaluation on heat Transfer Surfaces" Asme Journal Of Heat Transfer, 96, 2, pp. 16-21, 1975.

[12] L. Patiño, Análisis Teórico - Experimental de la Convección de Calor Transitoria en el Flujo de Fluidos a través de Medios Porosos. Tesis Presentada para optar al Título de Magister Scientiarum en Ingeniería Mecánica Mención Ciencias. Universidad de Oriente, Puerto La Cruz Venezuela, 2003.

[13] J. Bear, 'Dynamics Of Fluids In Porous Media'. American Elsevier Publishing Company, New York, 1976.

[14] L. Hunt and C. Tien, "Non Darcian convection in cilyndrical pocked beds", Asme Journal Of Heat Transfer. 10, 2, pp. 110-118, 1988.

[15] C. Maliska, 'Transferencia de Calor e Mecánica dos Fluidos Computacional". LTC Ed., Brasil, 1995.

[16] S. Patankar y D. Spalding, "A Calculation Procedure for Heat, Mass and Momentum Transfer in Three-Dimensional Parabolic Flows", Int. Journal Of Heat And Mass Transfer. 15, 7, pp. 1787-1806, 1972.

[17] S. Kline and F. McClintock, "Describing the Uncertainties in Single-Sample Experiments" Asme Mech Eng. 5, 1, pp. 3-8, 1953. 